\title{
Evolution of Microstructure during Rapid Solidification of SiC under High Pressure
}

\author{
Wanjun Yan $\mathbb{D}^{1,2}$ Xinmao Qin $\mathbb{D}^{1,{ }^{1,2}}$ Zhongzheng Zhang $\mathbb{D}^{1,2}$ Chunhong Zhang $\mathbb{D}^{1,2}$ \\ and Tinghong $\mathrm{Gao}^{3}$ \\ ${ }^{1}$ Electronic Information Engineering Institute, Anshun University, Anshun 561000, Guizhou, China \\ ${ }^{2}$ Fault Analysis and Reliability Research Integration Platform of Guizhou Province, Anshun University, Anshun 561000, \\ Guizhou, China \\ ${ }^{3}$ Institute of New Optoelectronic Materials and Technology, Guizhou University, Guiyan 550025, Guizhou, China
}

Correspondence should be addressed to Wanjun Yan; yanwanjun7817@163.com

Received 26 October 2021; Accepted 31 December 2021; Published 23 February 2022

Academic Editor: Maheswar Nayak

Copyright ( 2022 Wanjun Yan et al. This is an open access article distributed under the Creative Commons Attribution License, which permits unrestricted use, distribution, and reproduction in any medium, provided the original work is properly cited.

The microstructure evolution of liquid silicon carbide $(\mathrm{SiC})$ during rapid solidification under different pressure values is simulated with the Tersoff potential using molecular dynamics. The structure evolution characteristics of SiC are analyzed by considering the pair distribution function, bond angle distribution, coordination number, and the diagrams of the microstructure during rapid solidification. The results show that the average energy of atoms gradually increases with pressure. When the pressure reaches $100 \mathrm{GPa}$, the average energy of the atom is greater than the average energy of the atom in the initial liquid state. Under different pressures, the diffusion of atoms tends to remain stable at a temperature of about $3700 \mathrm{~K}$. The application of pressure has a major impact on the arrangement of atoms, except on the third-nearest neighbor, while the impact on the nearest neighbor and the second-nearest neighbor is relatively small. The pressure increases the medium-range order of the system. The coordination numbers of $\mathrm{Si}$ and $\mathrm{C}$ atoms gradually decrease with the decrease in temperature and increase in pressure. Pressure changes the microstructure of the $\mathrm{SiC}$ amorphous system after solidification, and the density can be increased by adjusting the coordination number of atoms. As the pressure increases, the $\mathrm{SiC}$ amorphous system exhibits a dense structure with coordination numbers of 4 , 5,6 , and 7 .

\section{Introduction}

Silicon carbide $(\mathrm{SiC})$ has excellent physical and mechanical properties, such as low density, high specific strength, and high-temperature resistance. It has great application prospects in fabricating weapon armor, coatings, engine turbine blades, and friction and electronic devices [1]. Extreme electronic devices, i.e., those based on wide-band-gap semiconductors, such as $\mathrm{SiC}, \mathrm{GaN}$-based nitride, and diamond, that are operated under complex and harsh conditions-such as at high temperatures and pressures, high strain rates, and even under strong radiation-are attracting substantial attention [2].

Extensive experimental research has been conducted to study the elastic deformation and phase transition of $\mathrm{SiC}$ under high pressures. Yoshida et al. [3] found that the 3C$\mathrm{SiC}$ structure transforms from sphalerite to rock salt at about $100 \mathrm{GPa}$ at room temperature (300K). Zhuravlev et al. [4] performed X-ray diffraction (XRD) and Raman experiments at room temperature. They reported that the $3 \mathrm{C}$-SiC lattice remains stable up to $75 \mathrm{GPa}$, and they also noted a tendency for destabilization above $40 \mathrm{GPa}$. Daviau and Lee [5] explored the stability of $3 \mathrm{C}-\mathrm{SiC}$ at high pressures and temperatures in a laser-heated diamond anvil cell. The threshold pressure for the phase transition from zincblende to rock salt structure was found to significantly decrease at high temperatures (1500 K and $1700 \mathrm{~K})$. Daviau and Lee [6] reported that the decomposition that can occur at high temperatures and pressures halted once the RS phase was formed. However, previous studies lacked effective dynamic in situ 
detection technology and could not identify the process of dynamic deformation of materials in real time. Recently, advanced in situ detection technology has been used worldwide to observe the coexistence of low- and highpressure phases in a mixed-phase system under laser shock, and the completion of the phase change has been found to require a high pressure of $>200 \mathrm{GPa}$ [7]. Nevertheless, the complete phase transition kinetics in real-time in situ conditions have still not been determined.

Molecular dynamics (MD) simulations have unique merits in revealing the atomic structure changes and micro-/ nano-scale deformations, and they have been successfully used in studying the mechanical properties [8], surface $[9,10]$, defects [11], crystal growth [12], and nanotubes and nanowires [13-16] of SiC. MD is especially suitable for the study of dynamic physical and mechanical property changes of materials under extreme loading $[17,18]$. Recently, Li et al. [19] used large-scale MD to simulate the elastic-plastic and structural phase transitions of $\mathrm{SiC}$ under compression loading. Results show that the plastic deformation and phase transition are strongly dependent on the strain rate.

Studying the influence of solidification process control on the preparation and performance of materials can form a more unified understanding of the relationship between the preparation process, structure, and performance. The study of the solidification process of materials has always been an important research topic in the field of materials science. Pressure and temperature are two important parameters that affect the solidification process of materials. The effect of temperature on the solidification process has been extensively studied $[20,21]$. In contrast, a few studies have explored the influence of pressure on the solidification process [22], especially the influence of high pressure on the solidification process, because there is no equipment that can directly observe the solidification process under highpressure conditions. $\mathrm{SiC}$ is one of the materials necessary for fabricating extreme electronic devices. The study of the rapid solidification process of $\mathrm{SiC}$ under high pressure is necessary. Therefore, the MD simulation method is used to simulate the rapid solidification process of $\mathrm{SiC}$ under different pressures and to study the influence of pressure on the dynamic behavior and microstructure of SiC. The evolution of the microstructure provides theoretical guidance for the study of the physical and mechanical properties of SiC under extreme service conditions and can be the basis of the design of related materials and structures.

\section{Simulation Conditions and Methods}

$\mathrm{SiC}$ composed of 8000 atoms is placed in a cubic box with periodic boundary conditions, and the Tersoff potential function [23] and Nosé-Hoover algorithm are used [24, 25] to control the temperature and pressure. The simulation time step is set to $1 \mathrm{fs}$, and the macro constraint condition is the constant-pressure, constant-temperature (NPT) ensemble. Firstly, the system is heated to a temperature of $4500 \mathrm{~K}$, which is higher than the melting point of $\mathrm{SiC}$, and 40,000 steps are isothermally run to obtain the liquid $\mathrm{SiC}$ at equilibrium. Then, at different pressures of $0,1,5,10,20,30$,
40, 50, and $100 \mathrm{GPa}$, the system is rapidly cooled from $4500 \mathrm{~K}$ to $100 \mathrm{~K}$ at a cooling rate of $1 \times 10^{12} \mathrm{~K} / \mathrm{s}$, and the atomic configuration is recorded for every $100 \mathrm{~K}$ decrement to analyze the microstructure.

\section{Results and Analysis}

3.1. Average Energy of Atoms and Volume Ratio. Figure 1 shows the average energy of atoms and the volume ratio as functions of temperature during the rapid solidification of the liquid $\mathrm{SiC}$ under different pressures.

Figure 1 shows that the volume ratio $\mathrm{V} / \mathrm{V}_{0}\left(\mathrm{~V}\right.$ and $\mathrm{V}_{0}$ are the liquid and solid volumes of $\mathrm{SiC}$, respectively) of liquid $\mathrm{SiC}$ during rapid solidification decreases with the increase in pressure. The average energy of atoms gradually increases with pressure, and the extent of change gradually becomes smaller. Furthermore, when the pressure reaches $100 \mathrm{GPa}$, the average energy of the atoms becomes greater than the average energy of the atoms in the initial liquid state. The reason for this change is that the interatomic distance in the liquid $\mathrm{SiC}$ system gradually reduces with the increase in pressure. Hence, the volume correspondingly decreases, and the value of $\mathrm{V} / \mathrm{V}_{0}$ becomes smaller. The distance reduces, resulting in a decrease in the mutual attraction potential energy between the atoms. As the temperature continues to decrease, the kinetic energy of the atoms also decreases. Therefore, during the solidification process, the average energy of the atoms shows a downward trend. When the applied pressure is $100 \mathrm{GPa}$, since the distance between atoms decreases and becomes smaller than the equilibrium distance $r_{0}$ between the atoms, the repulsive potential energy increases. Therefore, the average energy of atoms under high pressure of $100 \mathrm{GPa}$ is greater than that of liquids.

3.2. Mean Square Displacement (MSD). To analyze the influence of different pressures on the dynamics of the rapid solidification process, the relationship between the mean square displacement (MSD) and the temperature during the rapid solidification of liquid $\mathrm{SiC}$ was analyzed (Figure 2).

Figure 2 shows that during the rapid solidification of $\mathrm{SiC}$, as the pressure increases, MSD gradually decreases, and the slope of MSD also shows a decreasing trend, indicating that the diffusion coefficient of atoms decreases with the increase in pressure. The application of pressure hinders the movement of atoms. Under different pressures, the diffusion of atoms tends to remain stable at a temperature of about $3700 \mathrm{~K}$.

3.3. Radial Distribution Function (RDF). RDF is a function that describes the density of particles as a function of the distance from the reference atom. It is an important method to describe the ordering degree of liquid, crystalline, and amorphous structures. The formula of RDF is as follows:

$$
g(r)=\frac{d N}{\rho 4 \pi r^{2} d r} .
$$

To analyze the influence of pressure on the microstructure during the rapid solidification of $\mathrm{SiC}$, the curve of the radial 


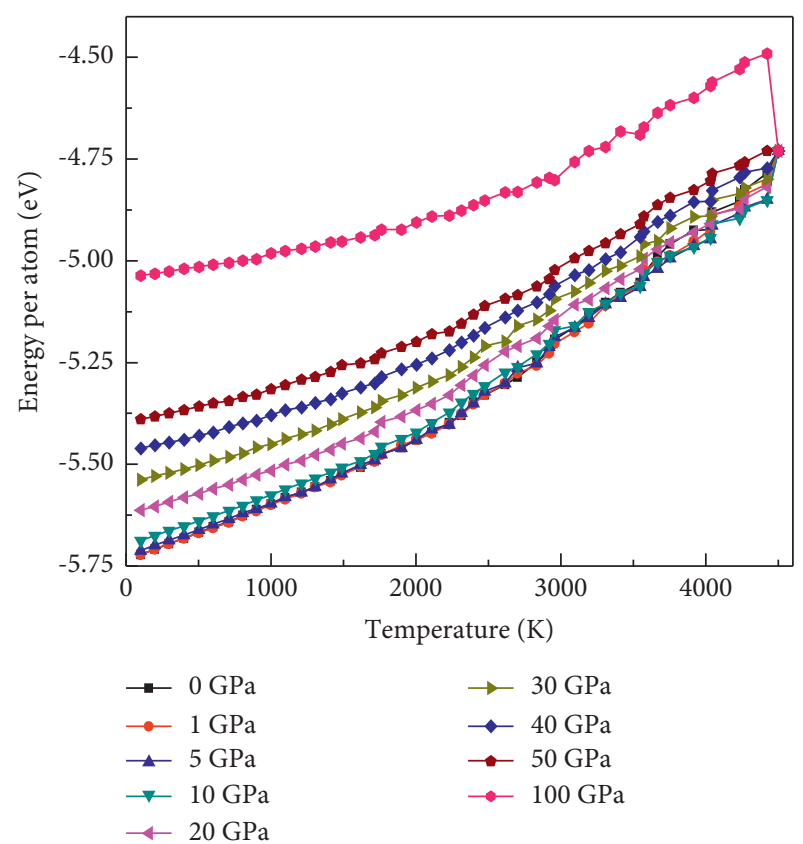

(a)

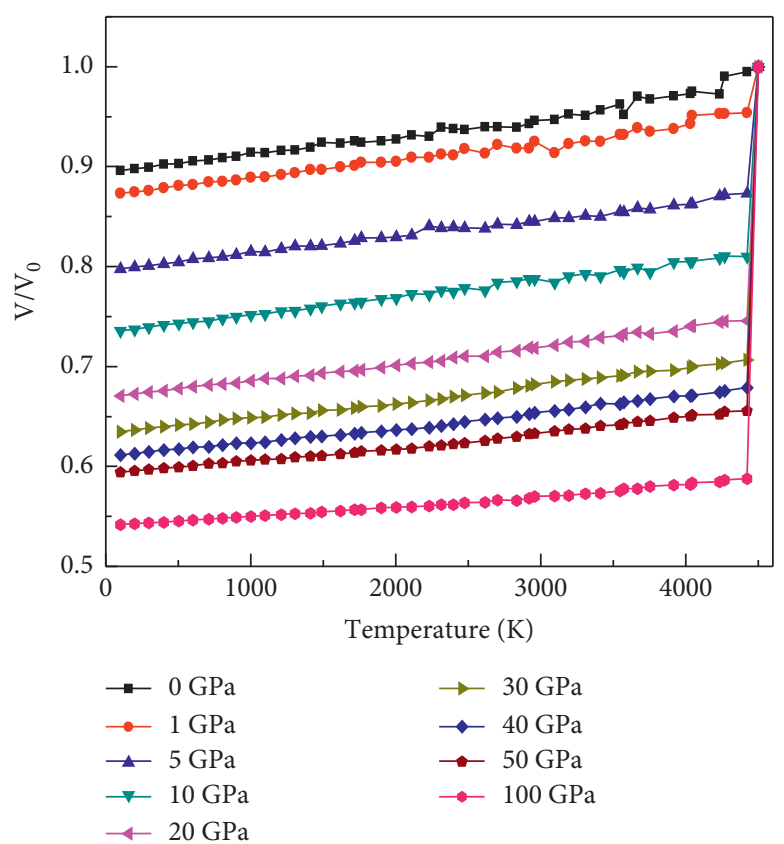

(b)

FIGURE 1: Average energy and volume ratio of atoms as functions of temperature during rapid solidification of SiC under different pressures.

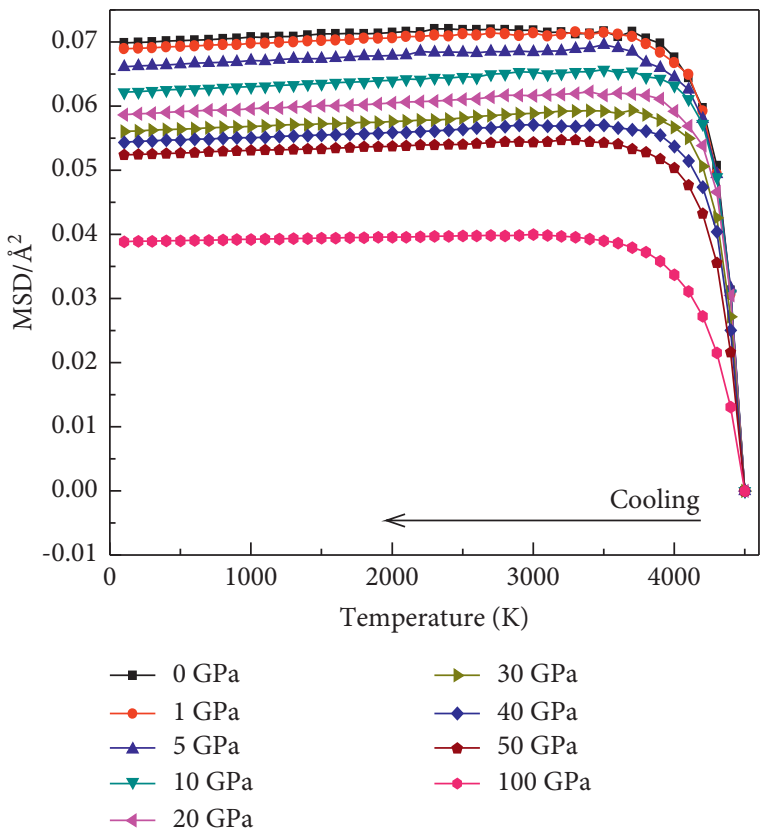

Figure 2: Relationship between MSD and temperature during rapid solidification of SiC under different pressures.

distribution function (RDF) as a function of temperature under different pressures (Figure 3 ) is considered.

Figure 3 shows that as the temperature decreases, the first and second peaks of RDF continue to rise and become sharp, indicating that the short-range order in the solidification process is enhanced when different pressures are applied. At the same temperature, the first peak near $1.50 \AA$ gradually decreases with increasing pressure. Two new peaks appear near $2.50 \AA$ and near $3.00 \AA$, and the peaks increased with increasing pressure, and the position of the peaks gradually decreased with decreasing temperature. Two small peaks near $2.85 \AA$ and $3.20 \AA$ decrease with increasing pressure. To clarify the composition of RDF peaks after solidification, the RDF after solidification of each bond of $\mathrm{SiC}$ under different pressures was calculated and analyzed, as shown in Figure 4.

Figures 4(b)-4(d) show that the first peak near $1.5 \AA$ corresponds to the $\mathrm{C}-\mathrm{C}$ bond, the second peak near $1.9 \AA$ 


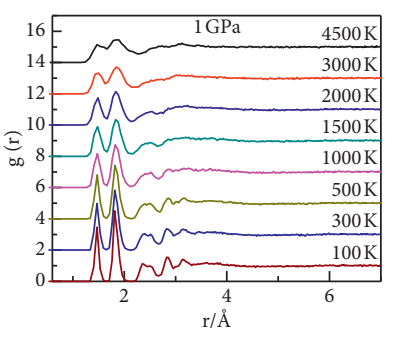

(a)

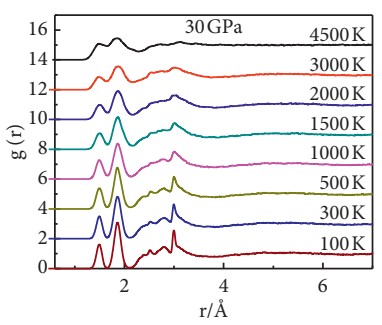

(e)

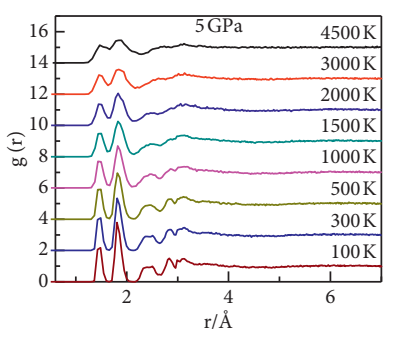

(b)

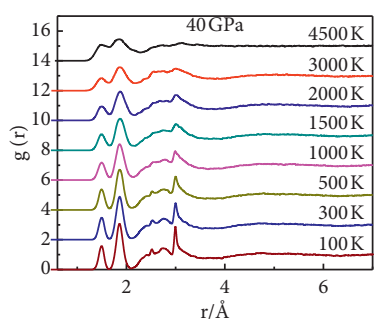

(f)

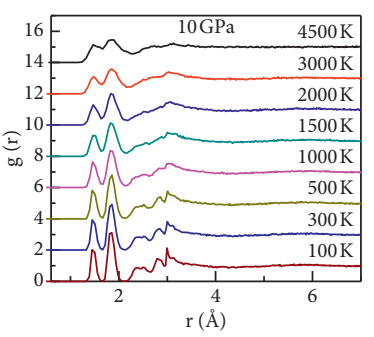

(c)

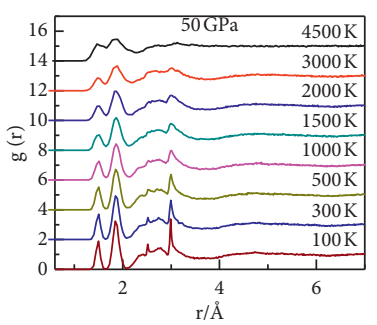

(g)

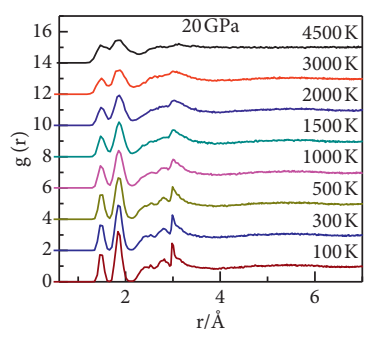

(d)

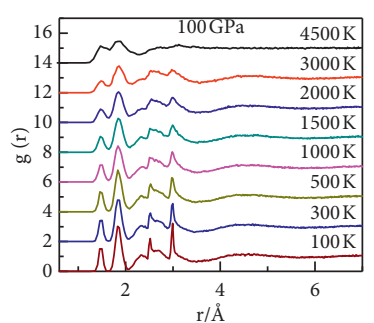

(h)

FIGURE 3: Change in $\mathrm{RDF}$ with the temperature of $\mathrm{SiC}$ under different pressures.

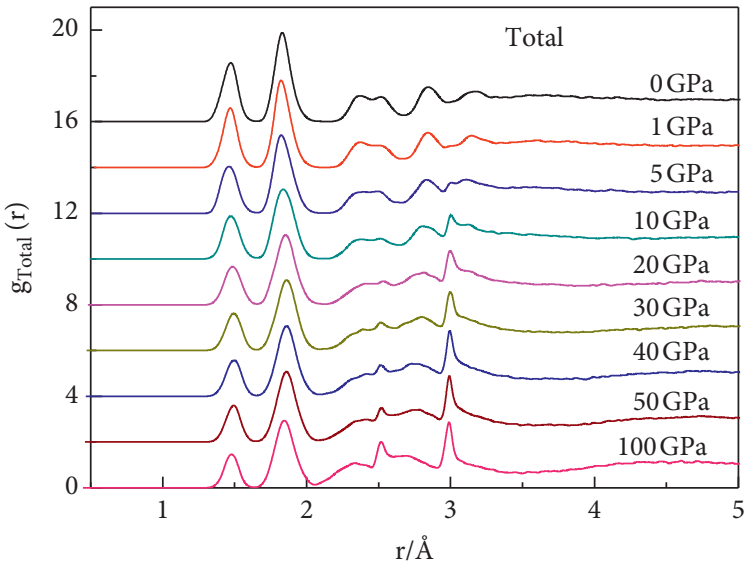

(a)

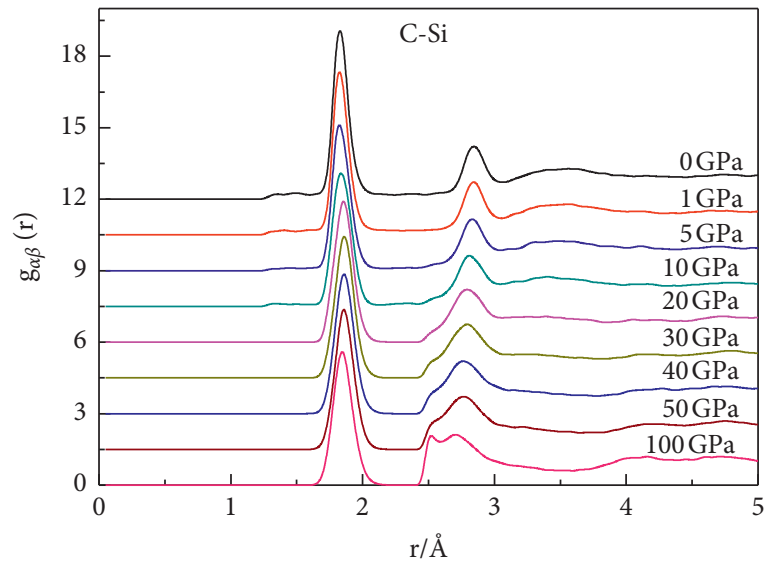

(c)

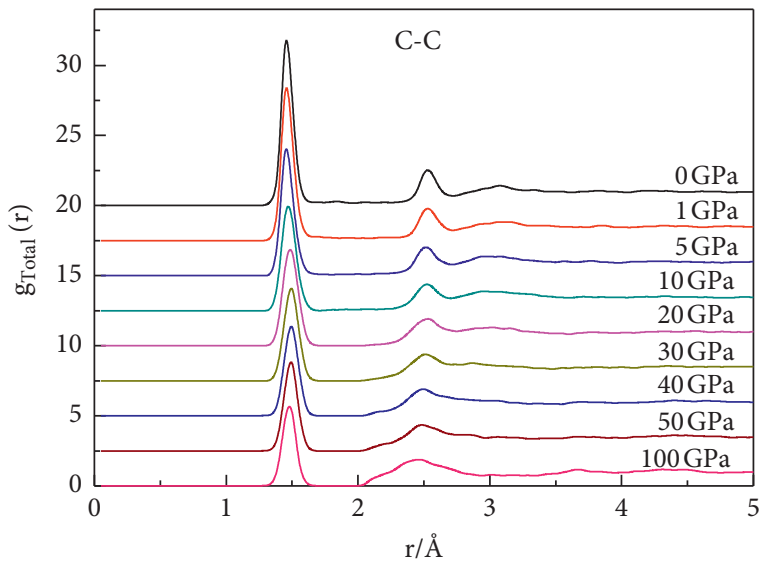

(b)

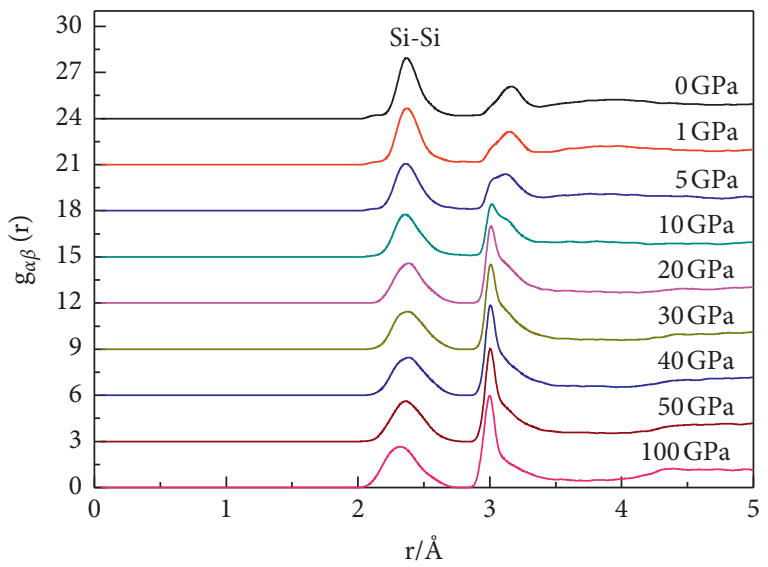

(d)

FIgURE 4: RDF after solidification of SiC under different pressures (100 K). 
corresponds to the $\mathrm{C}-\mathrm{Si}$ bond, and the third peak near $2.35 \AA$ corresponds to the $\mathrm{Si}-\mathrm{Si}$ bond. When the pressure is less than $20 \mathrm{GPa}$, the peak near $2.5 \AA$ is mainly ascribed to the $\mathrm{C}-\mathrm{C}$ bond, and the peak decreased as the pressure increased. When the pressure is greater than $20 \mathrm{GPa}$, a peak rises around $2.5 \AA$, which reflects that $\mathrm{C}-\mathrm{Si}$ atoms are replaced at their new positions to construct new short-range order. When the pressure is less than $10 \mathrm{GPa}$, the peak near $3.0 \AA$ corresponds mainly to the $\mathrm{C}-\mathrm{C}$ and $\mathrm{C}-\mathrm{Si}$ bonds, and the peak value increases with pressure. When the pressure is greater than $10 \mathrm{GPa}$, the second peak corresponding to the $\mathrm{Si}-\mathrm{Si}$ bond is compressed from $3.2 \AA$ to $3.0 \AA$. Therefore, this peak corresponded mainly to the $\mathrm{Si}-\mathrm{Si}$ bond, and its height increases significantly with pressure, while the peak near $3.2 \AA$ gradually decreased. From the above analysis, it can be seen that the application of pressure has a greater impact on the arrangement of atoms, except for the third-nearest neighbor, while the impact on the nearest neighbor and the secondnearest neighbor is relatively small.

The analysis of RDF in our previous study [26] showed that the $\mathrm{C}$ atoms distributed around $1.5 \AA$ mainly exhibited a coordination number of 3 . After applying pressure, the peak of RDF at $1.5 \AA$ decreases with the increase of pressure, which corresponds to the decrease in the number of three-coordinated $\mathrm{C}$ atoms, indicating that $\mathrm{C}$ atoms form other coordination structures. The peaks near $2.5 \AA$ and $3.0 \AA$ forward shift, and the increase corresponds to the increase in the number of atoms in this range, indicating that the increase in pressure makes the atomic arrangement of the system more compact and improves the medium-range order of the system.

3.4. Coordination Number $(\mathrm{CN})$. The coordination number (CN) reflects the arrangement of the nearest-neighbor atoms, and it is a statistical parameter used for studying the arrangement of the nearest-neighbor atoms. Figure 5 shows the variation in the $\mathrm{CN}$ of $\mathrm{Si}$ and $\mathrm{C}$ atoms with temperature under different pressures.

It can be seen from Figure 5(a) that during the rapid solidification of liquid $\mathrm{SiC}$, the $\mathrm{CN}$ of all $\mathrm{Si}$ atoms is greater than four. When the pressure is higher than $1 \mathrm{GPa}$, the $\mathrm{CN}$ of $\mathrm{Si}$ atoms gradually decreases with decreasing temperature and increases with increasing pressure. When the pressure reaches $100 \mathrm{GPa}$, the $\mathrm{CN}$ of $\mathrm{Si}$ atoms exceeds six. It can be seen from Figure 5(b) that when the pressure is less than $5 \mathrm{GPa}$, the $\mathrm{CN}$ of $\mathrm{C}$ atoms increases with the decrease in temperature. When the pressure is greater than $5 \mathrm{GPa}$, the $\mathrm{CN}$ of $\mathrm{C}$ atoms increases with increasing pressure and gradually decreases with decreasing temperature when the pressure is greater than $40 \mathrm{GPa}$. The $\mathrm{CN}$ of $\mathrm{C}$ atoms in the solidified $\mathrm{SiC}$ is greater than four, indicating that as the pressure increases, the $\mathrm{C}$ atoms no longer form a three-coordinated structure.

3.5. Bond Angle Distribution Function (BADF). The bond angle distribution function (BADF) reflects the positional relationship between the neighboring atoms and the central atom and provides information about the structural evolution of the system during solidification under different pressures. Figure 6 shows the BADF of $\mathrm{SiC}$ crystal at different temperatures under different pressures.

Figure 6 shows that the main peaks become higher and narrower as the temperature decreases. When the pressure is $1 \mathrm{GPa}$, the main peak is at around $120^{\circ}$, and the bond angles are mainly distributed between $70^{\circ}$ and $170^{\circ}$. When the pressure is $5 \mathrm{GPa}$, the main peak at $120^{\circ}$ decreases, and another peak starts to appear at $110^{\circ}$. As the pressure increases to $10 \mathrm{GPa}$, a new peak appears near $60^{\circ}$, the $120^{\circ}$ peak further decreases, and the $110^{\circ}$ peak's height increases. Furthermore, when the pressure is $20 \mathrm{GPa}$, the $110^{\circ}$ peak is higher than that of $120^{\circ}$. A new peak appears at $80^{\circ}$, and a shoulder peak appears in the range of $140^{\circ}$ to $180^{\circ}$. With a further increase in pressure, three main peaks are formed near $60^{\circ}, 80^{\circ}$, and $110^{\circ}$. The range of the shoulder peak and the bond angle distribution also increase with pressure, and the bond angle distribution range becomes $40^{\circ}$ to $180^{\circ}$ at $100 \mathrm{GPa}$.

To analyze the influence of pressure on the microstructure of liquid $\mathrm{SiC}$ after solidification, the bond angle distribution function at $100 \mathrm{~K}$ and under different pressures was analyzed (Figure 7).

Figure 7 shows that in the pressure range of $0-10 \mathrm{GPa}$, the bond angles after the solidification of $\mathrm{SiC}$ are mainly distributed around $120^{\circ}$ and $110^{\circ}$, indicating that an amorphous random network structure dominated by threeand four-coordinated structures is formed after solidification. With the increase in pressure, the BADF around $120^{\circ}$ gradually decreased, indicating that the number of threecoordinated structures decreases with the increase of pressure. The results of the analysis of the change in $\mathrm{CN}$ with pressure show that the $\mathrm{CN}$ of the $\mathrm{C}$ atom increases with pressure, i.e., the three-coordinated structure of $\mathrm{C}$ gradually changes to the four-coordinated structure, and the number of the four-coordinated structure increases, which is corresponding to the increasing of $\mathrm{BADF}$ around $110^{\circ}$. In the pressure range of $20 \mathrm{GPa}$ to $100 \mathrm{GPa}$, the BADF around $60^{\circ}$, $80^{\circ}, 110^{\circ}$, and $140^{\circ}$ to $180^{\circ}$ increases with pressure, indicating that a new coordination structure is formed in the system.

Figure 8 shows the coordination of $\mathrm{C}$ and $\mathrm{Si}$ atoms and their bond angle distribution. For example, cn3_C and cn4_C represent the three-coordinated bond angle and the four-coordinated bond angle, respectively, with the $\mathrm{C}$ atom at the center, and so on.

It can be seen from Figure 8(a) that the bond angle of the three-coordinated $\mathrm{C}$ atom is mainly distributed around $120^{\circ}$, and the peak value gradually decreases with increasing pressure. Figure 8 (b) shows that the number of four-coordinated $\mathrm{C}$ atoms increases with increasing pressure. It can be seen from Figure 8(c) that the bond angles corresponding to the four-coordinated $\mathrm{Si}$ atoms are mainly between $70^{\circ}$ and $140^{\circ}$, and the number decreases with increasing pressure. At $100 \mathrm{GPa}$, the number of four-coordinated Si atoms is almost zero. It can be seen from Figure 8(d) that the bond angles corresponding to the five-coordinated $\mathrm{Si}$ atoms are mainly distributed between $70^{\circ}$ and $180^{\circ}$, and the number of fivecoordinated $\mathrm{Si}$ atoms increases with pressure $(0-30 \mathrm{GPa})$. In the range of $30 \mathrm{GPa}$ to $100 \mathrm{GPa}$, the number of five-coordinated $\mathrm{Si}$ atoms decreases with increasing pressure. Figure 8(e) shows that when the pressure is higher than 


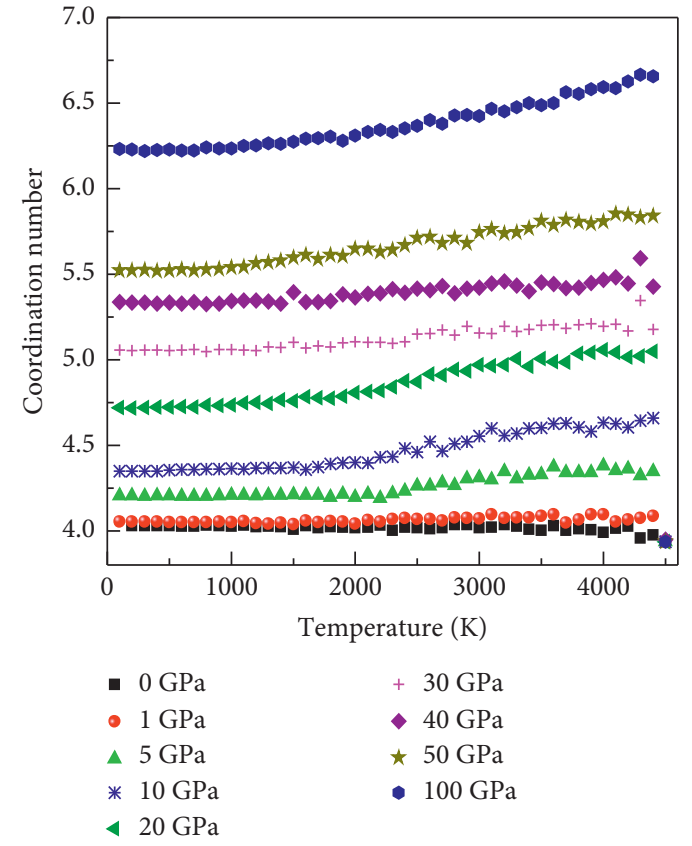

(a)

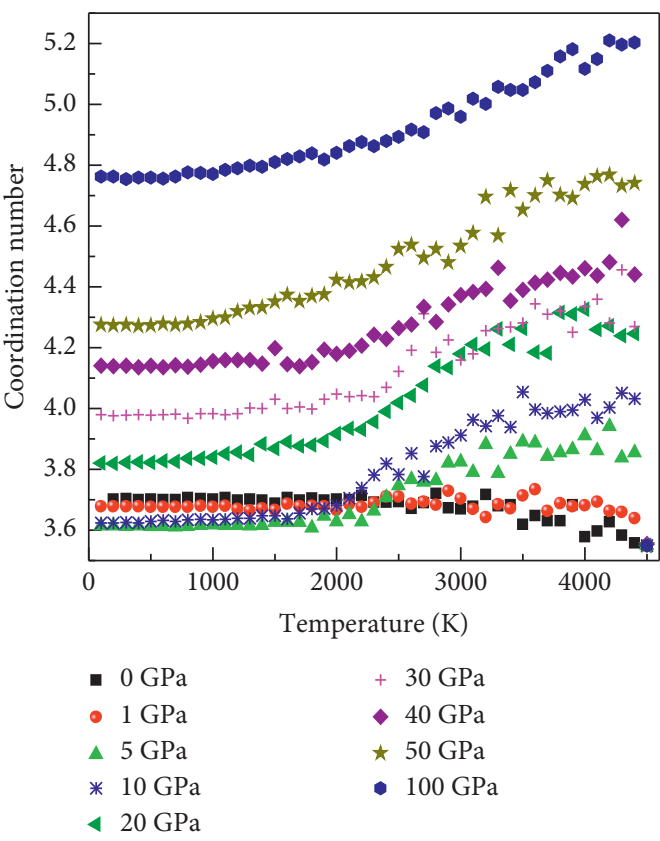

(b)

Figure 5: Change in the $\mathrm{CN}$ of $\mathrm{Si}$ and $\mathrm{C}$ atoms with temperature under different pressures.

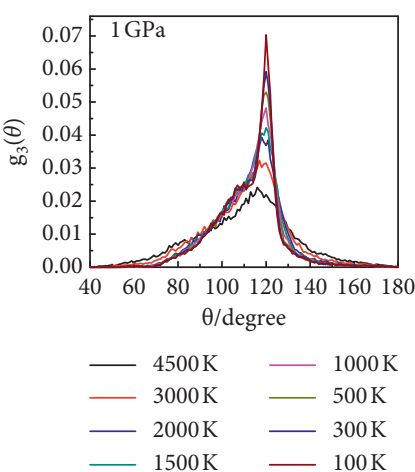

(a)
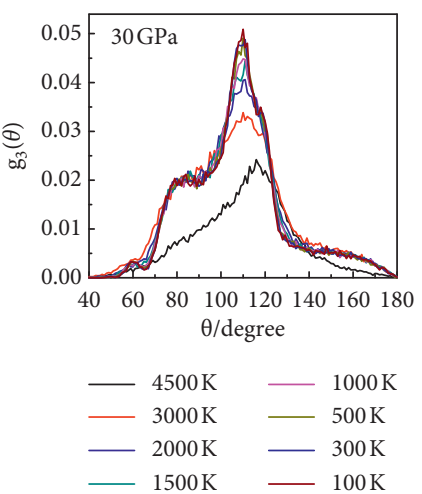

(e)
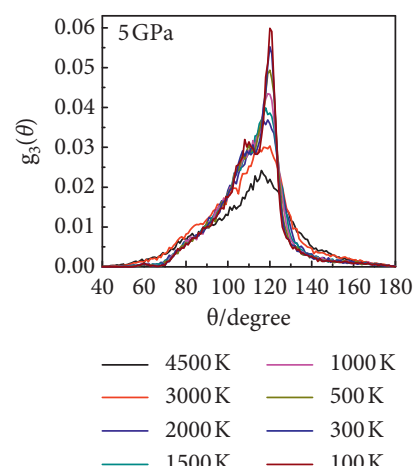

(b)
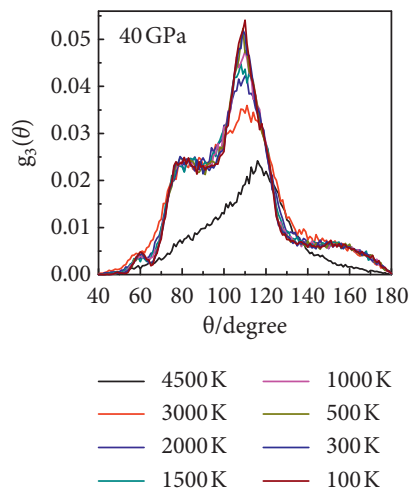

(f)
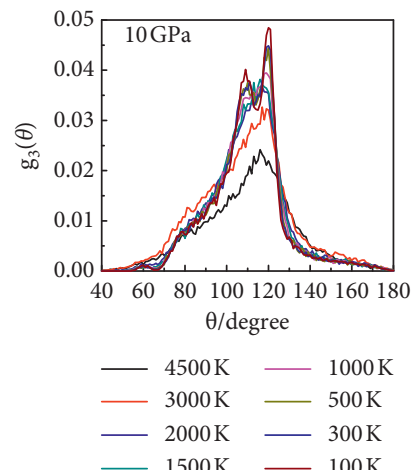

(c)
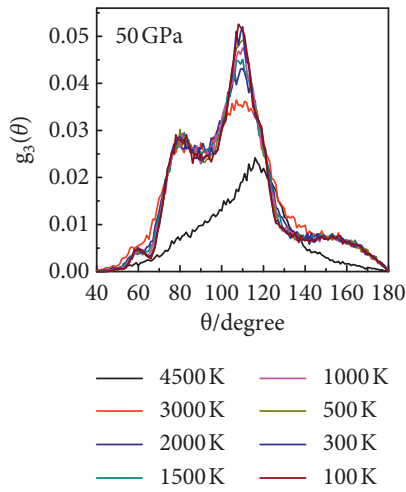

(g)
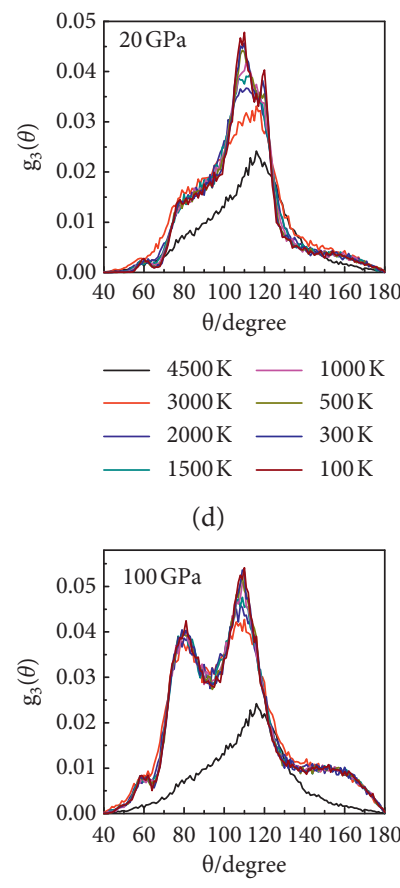

$-4500 \mathrm{~K}-1000 \mathrm{~K}$

$-3000 \mathrm{~K}-500 \mathrm{~K}$

$-2000 \mathrm{~K}-300 \mathrm{~K}$

- $1500 \mathrm{~K}-100 \mathrm{~K}$

(d)

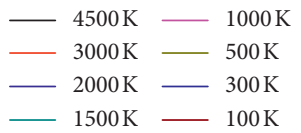

(h)

FIgURE 6: Change in BADF of SiC with temperature under different pressures. 


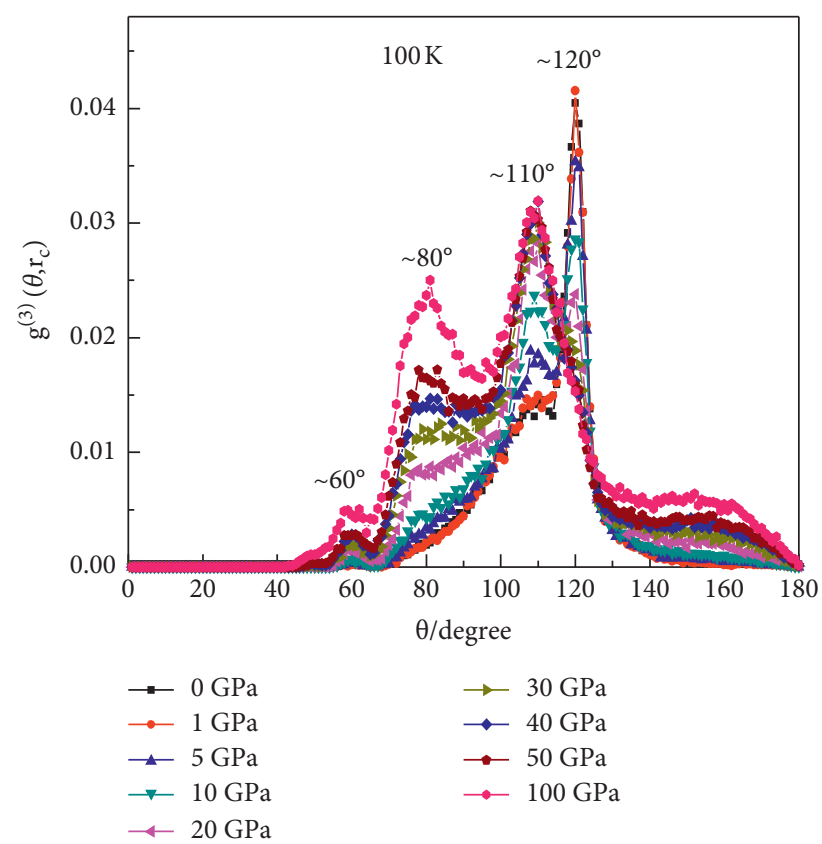

Figure 7: BADF of $\mathrm{SiC}$ after solidification under different pressures.
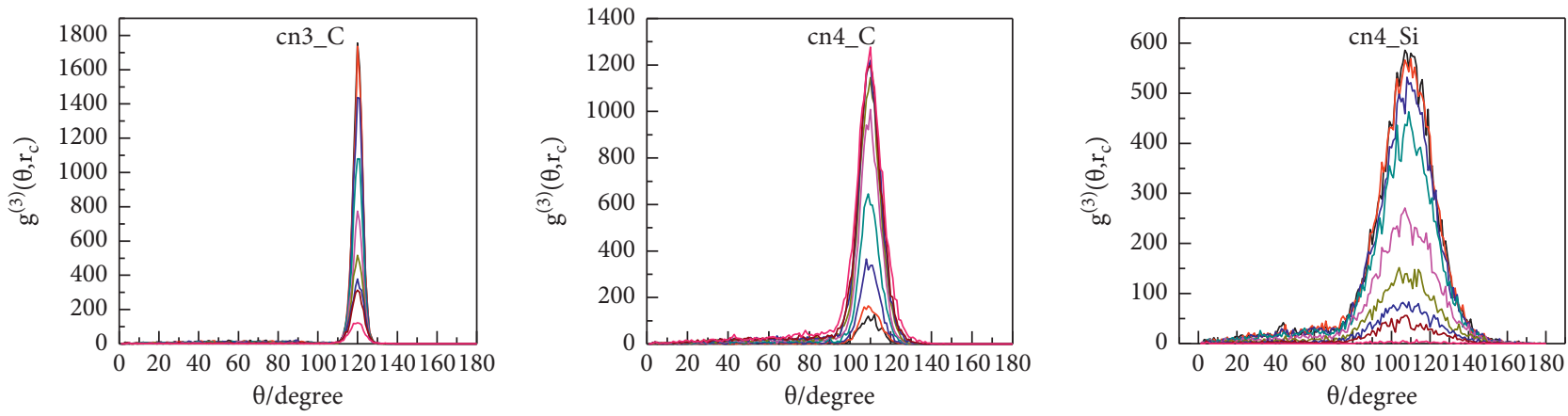

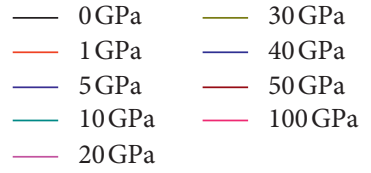

(a)
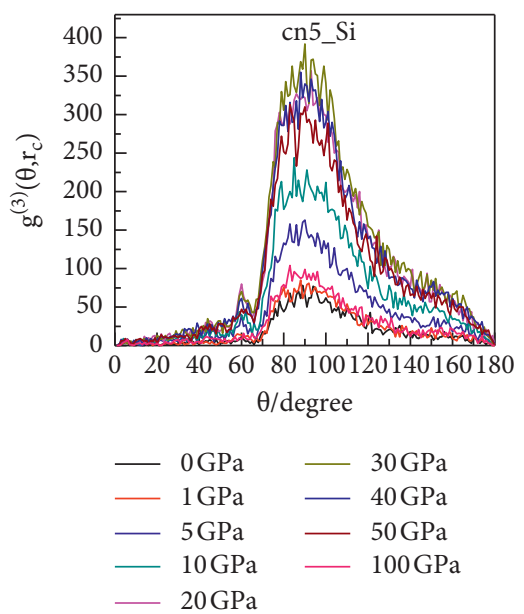

(d)

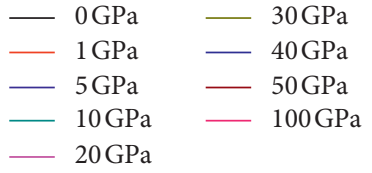

(b)
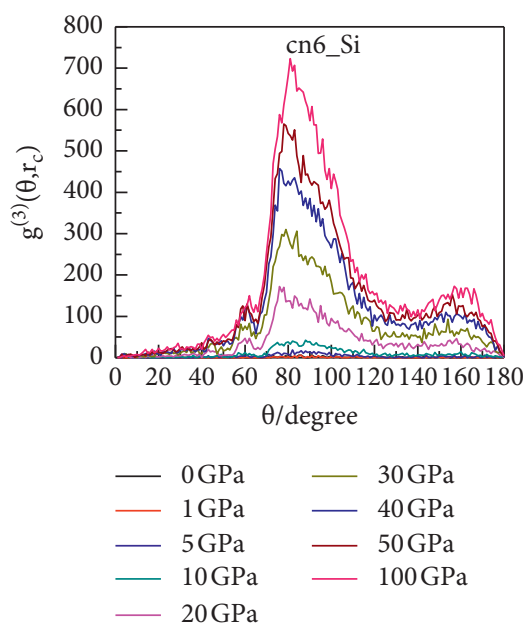

(e)

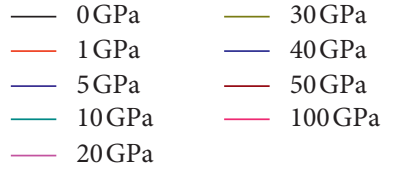

(c)

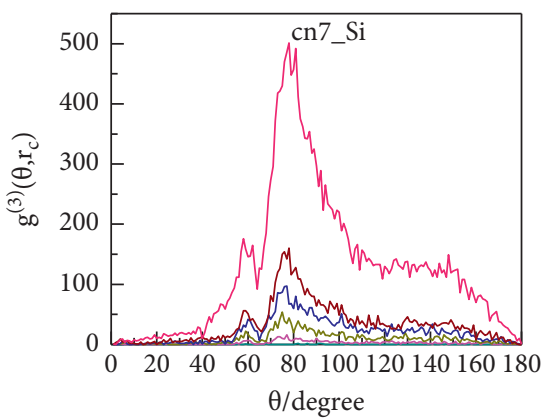

Figure 8: At $100 \mathrm{~K}$, the relationship between the number of bond angles centered on Si and C atoms with pressure. 
$20 \mathrm{GPa}$, the bond angles corresponding to the six-coordinated $\mathrm{Si}$ atoms are mainly between $40^{\circ}$ and $180^{\circ}$, and there is a strong peak near $80^{\circ}$ and another peak between $130^{\circ}$ and $170^{\circ}$. The number of plateaus increases with pressure. It can be seen from Figure 8(f) that the bond angles corresponding to the seven-coordinated $\mathrm{Si}$ atoms are mainly between $40^{\circ}$ and $180^{\circ}$. There are two obvious peaks near $60^{\circ}$ and $80^{\circ}$, and there is a plateau between $110^{\circ}$ and $150^{\circ}$. The peak value increases with pressure.

In summary, the pressure changes the microstructure of the $\mathrm{SiC}$ amorphous system after solidification, and the density is increased as a result of the changes in $\mathrm{CN}$ atoms. As the pressure increases, the $\mathrm{SiC}$ amorphous system exhibits a dense structure with a CN of $4,5,6$, and 7 .

\subsection{Microstructure Analysis}

3.6.1. Voronoi Polyhedron. The above analysis results show that during the solidification of liquid $\mathrm{SiC}$, the pressure has an impact on the nearest neighbor, second-nearest neighbor, third-nearest neighbor, and on the $\mathrm{CN}$ and bond angle distribution. To identify the effect of pressure on the arrangement of atoms, the Voronoi polyhedron method [27] was used to analyze the number of Voronoi polyhedrons during the solidification process. The Voronoi index method is represented by $(\mathrm{n} 3, \mathrm{n} 4, \mathrm{n} 5, \mathrm{n} 6)$, where $\mathrm{n} 3, \mathrm{n} 4, \mathrm{n} 5$, and $\mathrm{n} 6$ represent the number of triangles, quadrilaterals, pentagons, and hexagons in the polyhedron, respectively. For example,

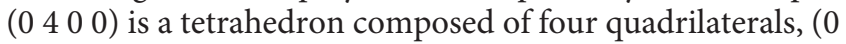
$600)$ is a hexahedron composed of six quadrilaterals, and (2 300 ) is a pentahedron composed of two triangles and three quadrilaterals. The minimum $\mathrm{CN}$ in the Voronoi index method is four, which corresponds to tetrahedral clusters. This method can accurately describe the crystalline and amorphous structures of semiconductor materials.

Figure 9 shows the large number of clusters that appeared during the solidification process.

Figure 10 shows the variation in the number of Voronoi polyhedrons with temperature under different pressures.

It can be seen from Figure 10 that when the pressure is $1-100 \mathrm{GPa}$, as the temperature decreases, the $\left(\begin{array}{llll}4 & 0 & 0 & 0\end{array}\right)$ structure in the system gradually increases, however, the trend of change in the number of $\left(\begin{array}{llll}4 & 0 & 0 & 0\end{array}\right)$ structures with the increase in the pressure is different. When the pressure is $1-20 \mathrm{GPa}$, the number of ( $\left.\begin{array}{llll}4 & 0 & 0 & 0\end{array}\right)$ structures increases with increasing pressure. Furthermore, for pressure between $20-50 \mathrm{GPa}$, the number of $\left(\begin{array}{llll}4 & 0 & 0 & 0\end{array}\right)$ structures decreases with increasing pressure, and the

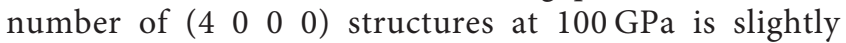
increased than that of $50 \mathrm{GPa}$. When the pressure is

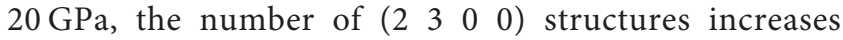
slowly with decreasing temperature, while that of $\left(\begin{array}{llll}0 & 6 & 0 & 0\end{array}\right)$ structure decreases with increasing temperature. When the pressure is $30-50 \mathrm{GPa}$, the number of (2 3000$)$ structures increases with the decrease in temperature and decreases with the increase in pressure. At $30 \mathrm{GPa}$, the number of (2 3000$)$ structures is the largest. The number of $\left(\begin{array}{llll}0 & 6 & 0 & 0\end{array}\right)$ structures increases with decreasing temperature and increases with increasing pressure. At

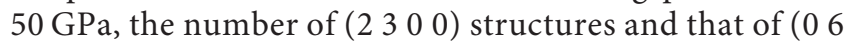
$00)$ structures in the system are almost the same. When the pressure increases to $100 \mathrm{GPa}$, the number of (2 300 ) structures is greatly reduced, and the number of $\left(\begin{array}{llll}0 & 6 & 0 & 0\end{array}\right)$ structures and that of (lllll $\left.\begin{array}{llll}0 & 5 & 2 & 0\end{array}\right)$ structures exceed the

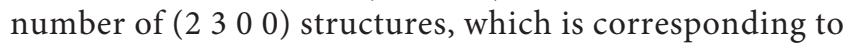
the reduction of the number of five-coordination atomic bond angles. During the high-pressure solidification process, the number of types of Voronoi polyhedrons increases with pressure, resulting in a complex structure with high coordination. The greater the pressure, the more coordinated the Voronoi polyhedrons, and the more complex the structure.

3.6.2. Cross-Section Atomic Distribution. To visually present the influence of pressure on the microstructure of amorphous $\mathrm{SiC}$ after solidification, partial cross-sectional views of the amorphous $\mathrm{SiC}$ system at $100 \mathrm{~K}$ after rapid solidification under different pressures are drawn, as shown in Figure 11: the crosssectional thickness is 1.5 atomic layers. It can be seen from the figure that the rapid solidification of the system under different pressures results in the formation of an amorphous structure without periodical translation characteristics. With the increase in pressure, the number of bonds between the atoms is different. When the pressure is $0-5 \mathrm{GPa}$, the atoms in the cross-section are partially bonded to form large rings, and some atoms are far apart and scattered in the system. When the pressure is increased to $10 \mathrm{GPa}$, the system structure becomes denser with some medium rings, and the number of scattered atoms decreases compared with the situation of $5 \mathrm{GPa}$. With a further increase in pressure, the number of rings in the system gradually increases, and the cavities formed by the rings become smaller. A three-membered ring appears in the system at $20 \mathrm{GPa}$. When the pressure is increased to $100 \mathrm{GPa}$, the density of the system increases significantly, and the number of three-membered rings and that of four-membered rings increase. Except for a few atoms, most of the atoms are bonded, which improves the medium-range order of the system. These results correspond to the results of the analysis based on $\mathrm{RDF}$, in which the influence of pressure on the degree of medium-range order was investigated.

3.6.3. Distribution of Central Atoms with Different CN. According to the results of the analysis of the bond angle distribution function, at high pressure, the $\mathrm{SiC}$ amorphous system exhibits a dense structure with $\mathrm{CN}$ of $4,5,6$, and 7 . Figure 12 shows the pressure-dependent changes in the number of central atoms with different $\mathrm{CN}$ in the amorphous $\mathrm{SiC}$ solidified under different pressures.

It can be seen from Figure 12 that in the pressure range of $5-100 \mathrm{GPa}$, the number of four-coordinated atoms is always the largest. Over the entire pressure range studied, the number of three-coordinated atoms decreases sharply with the increase in pressure, and the magnitude of the decrease decreases with the increase in pressure. Furthermore, the number of five-coordinated atoms varies with pressure in the range of $0-30 \mathrm{GPa}$, however, at 


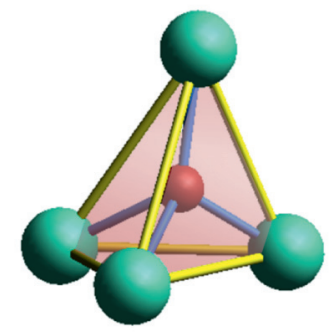

$\left(\begin{array}{llll}4 & 0 & 0 & 0\end{array}\right)$

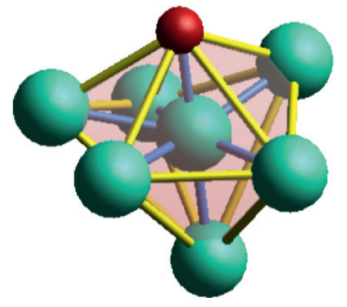

(0 $\left.5 \begin{array}{lll} & 2 & 0\end{array}\right)$

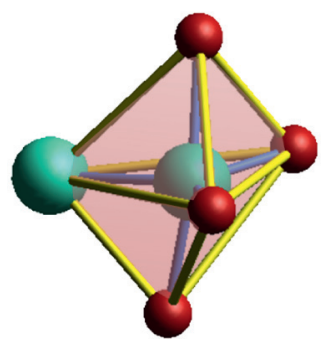

$\left(\begin{array}{llll}2 & 3 & 0 & 0\end{array}\right)$

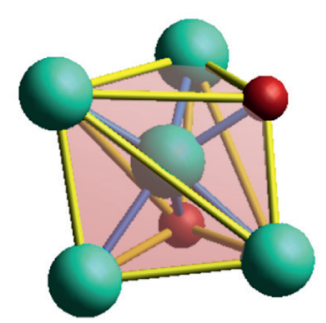

$\left(\begin{array}{llll}2 & 2 & 2 & 0\end{array}\right)$

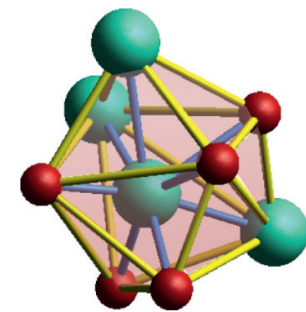

(0 4440$)$

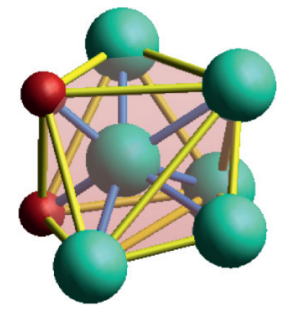

$\left(\begin{array}{llll}1 & 3 & 3 & 0\end{array}\right)$

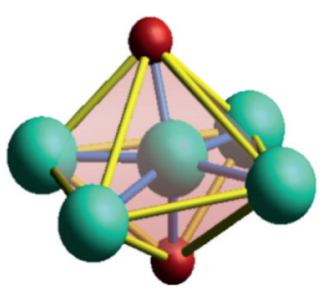

$\left(\begin{array}{llll}0 & 6 & 0 & 0\end{array}\right)$

FIGURE 9: Basic clusters appearing during solidification of liquid SiC.
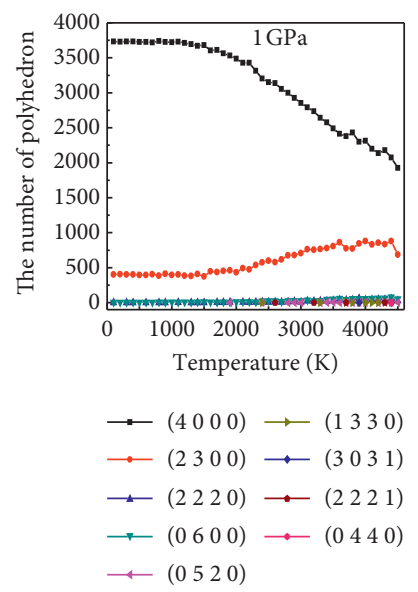

(a)

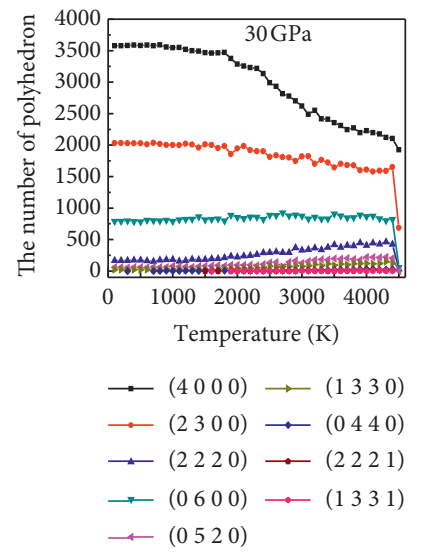

(e)

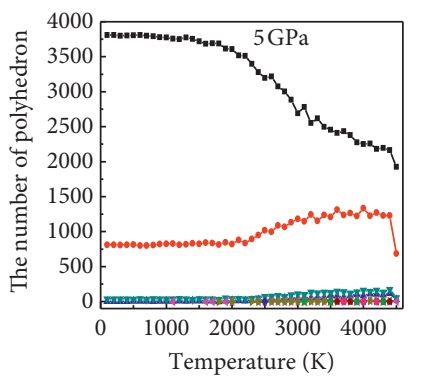

$\longrightarrow\left(\begin{array}{lllll}4 & 0 & 0 & 0\end{array}\right) \longrightarrow\left(\begin{array}{llll}1 & 3 & 3 & 0\end{array}\right)$

$\longrightarrow(23000) \rightarrow\left(\begin{array}{llll}3 & 0 & 3 & 1\end{array}\right)$

$\longrightarrow(2220) \longrightarrow(22221)$

$\longrightarrow$ (0 6000$) \longrightarrow\left(\begin{array}{llll}1 & 3 & 3 & 1\end{array}\right)$

$\longleftarrow(0520)$ « (0440)

(b)

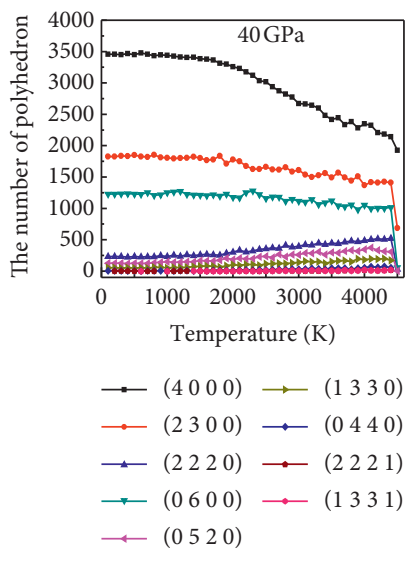

(f)

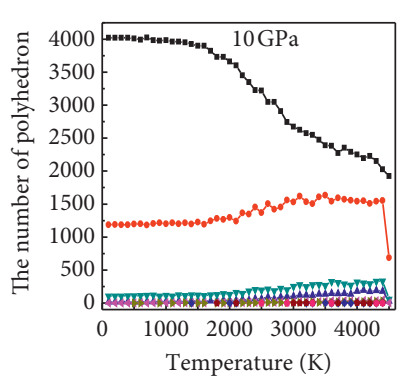

$\longrightarrow\left(\begin{array}{lllll}4 & 0 & 0 & 0\end{array}\right) \longrightarrow$ (1 3330$)$

$\longrightarrow(2300) \longrightarrow(0440)$

$\longrightarrow(2220) \longrightarrow(22221)$

$\rightarrow\left(\begin{array}{llll}0 & 6 & 0 & 0\end{array}\right) \longrightarrow\left(\begin{array}{llll}1 & 3 & 3 & 1\end{array}\right)$

$\longleftarrow(0520)$

(c)

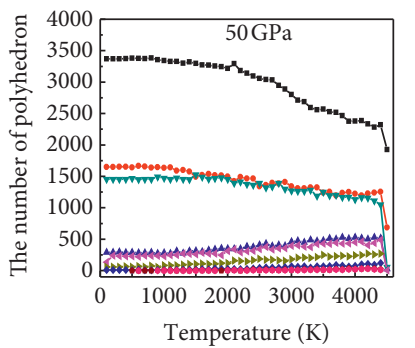

$\longrightarrow\left(\begin{array}{llll}4 & 0 & 0 & 0\end{array}\right) \rightarrow\left(\begin{array}{llll}1 & 3 & 3 & 0\end{array}\right)$

$\longrightarrow(23300) \longrightarrow(04440)$

$\rightarrow(22220) \rightarrow\left(\begin{array}{llll}1 & 3 & 3 & 1\end{array}\right)$

$\rightarrow\left(\begin{array}{llll}0 & 6 & 0 & 0\end{array}\right) \longrightarrow\left(\begin{array}{llll}2 & 2 & 2 & 1\end{array}\right)$

$\leftarrow\left(\begin{array}{llll}0 & 5 & 2 & 0\end{array}\right)$

(g)

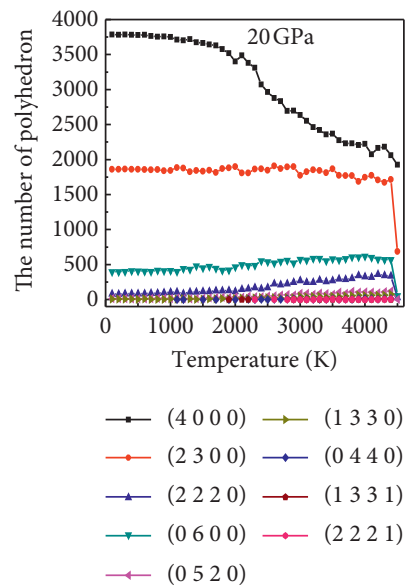

(d)

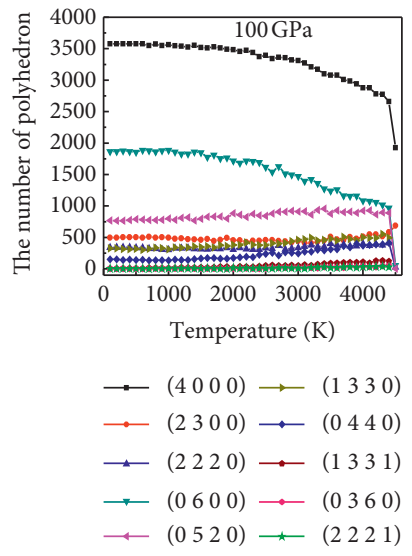

(h)

Figure 10: Variation in the number of Voronoi polyhedrons with temperature under different pressures. 


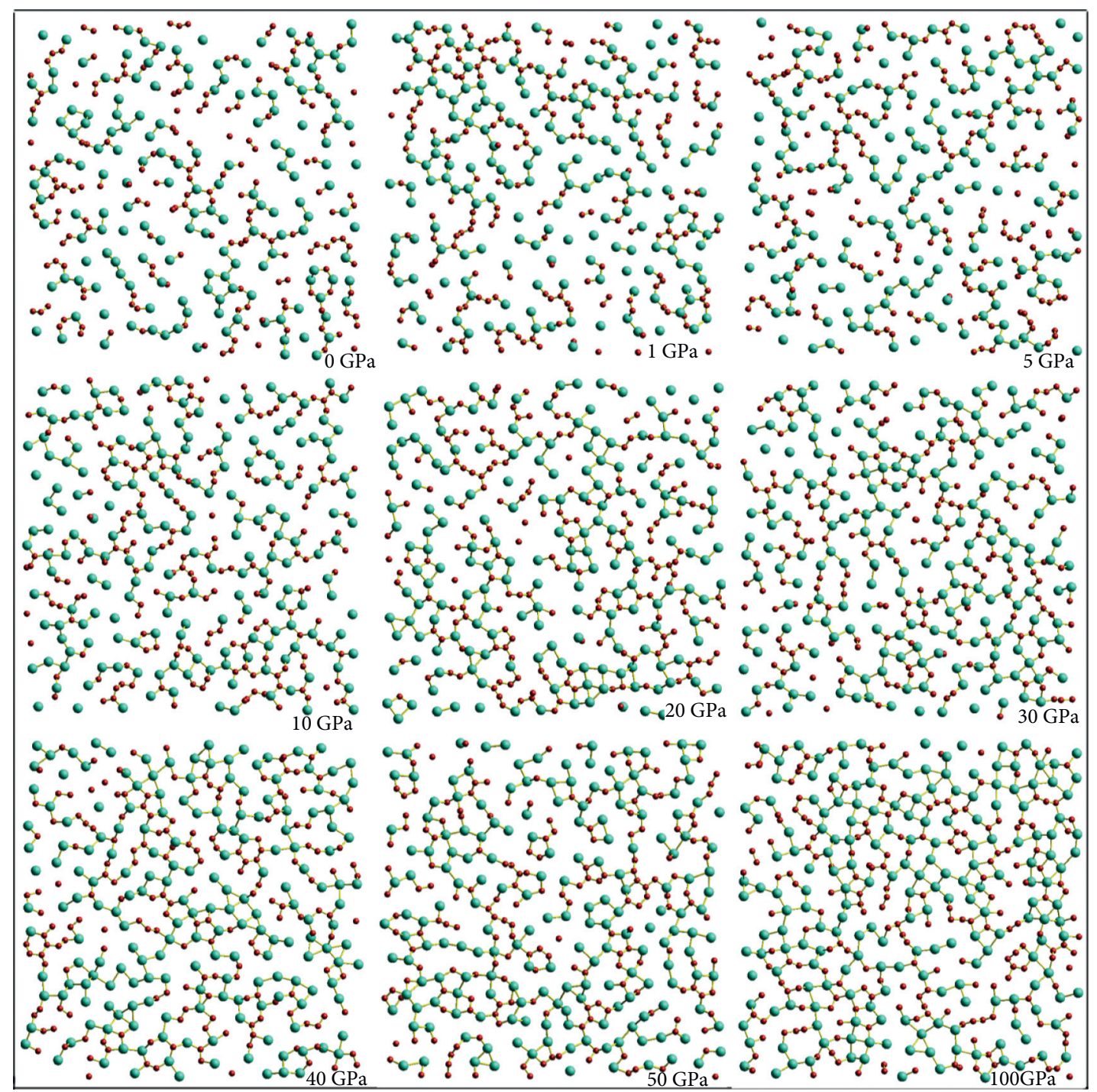

FIgURE 11: Partial cross-section of amorphous SiC after solidification under different pressures and at $100 \mathrm{~K}$ (thickness of 1.5 atomic layers).

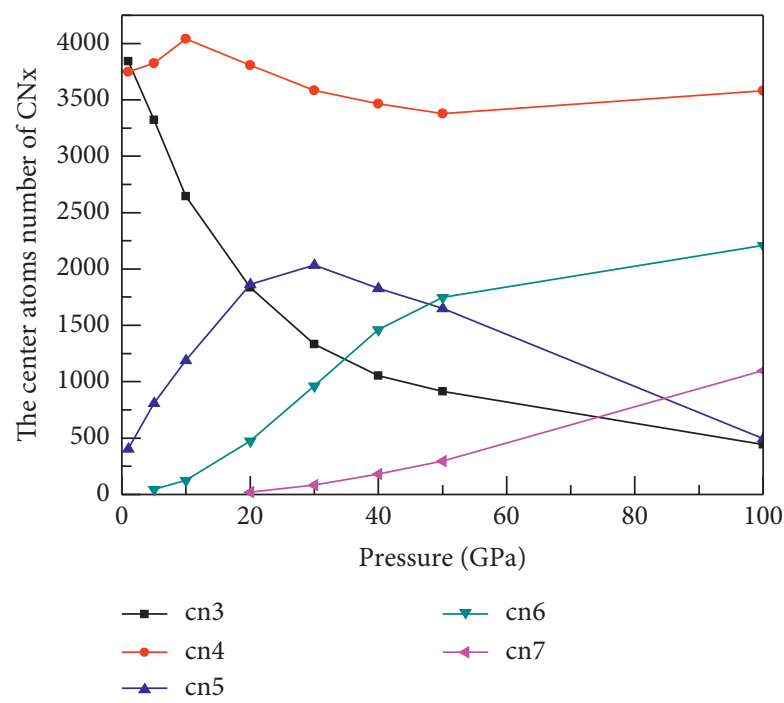

Figure 12: Pressure-dependent variation in the number of central atoms with different CN in amorphous SiC (100 K). 

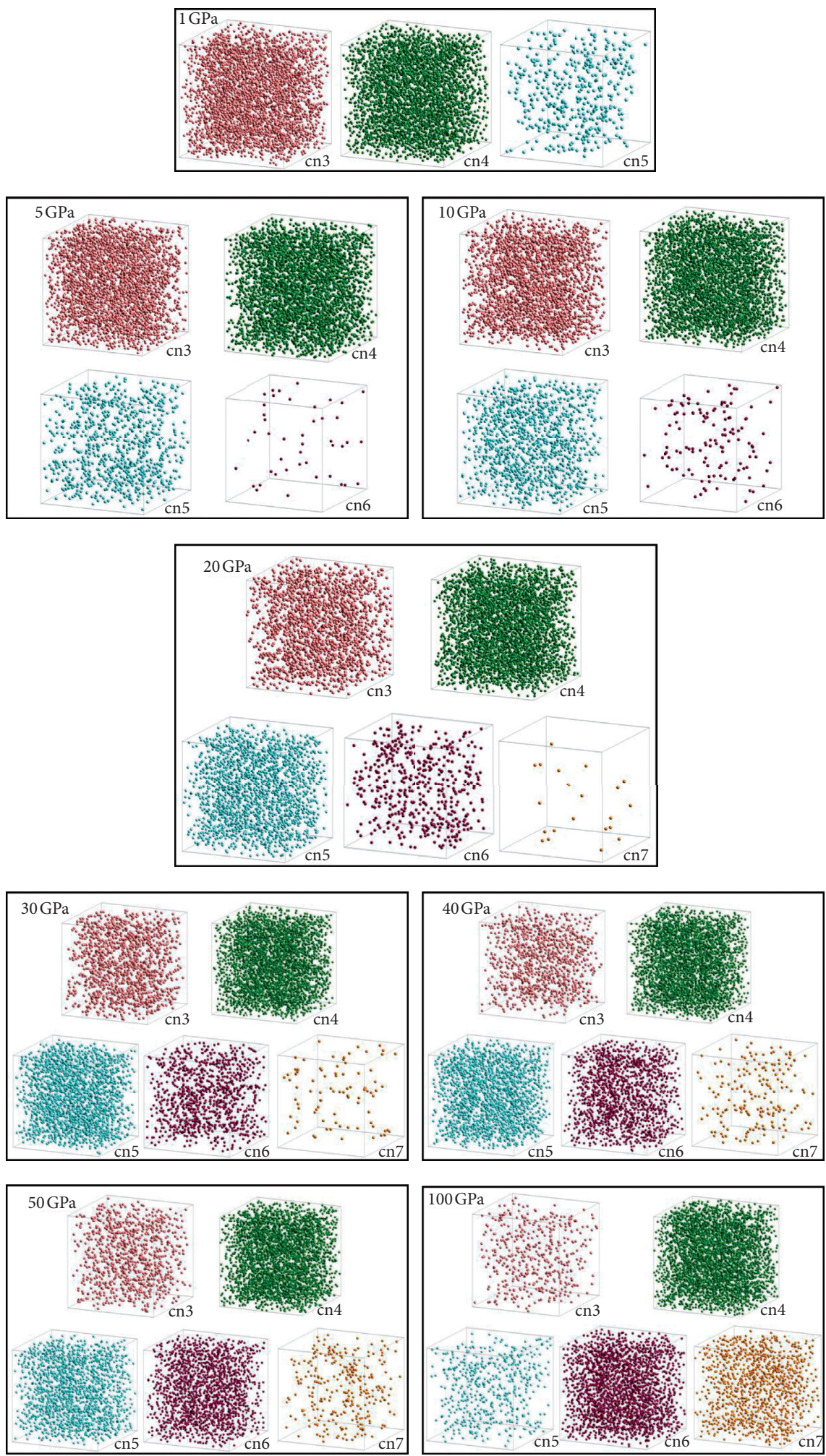

FIgURE 13: Distribution of central atoms with different $\mathrm{CN}$ in amorphous $\mathrm{SiC}$ system $(100 \mathrm{~K})$.

pressures higher than $30 \mathrm{GPa}$, it decreases with increasing pressure. The number of atoms of $\mathrm{CN}$ of six and seven increases with pressure. These results agree with the pressure-dependent variation in $\mathrm{CN}$. Figure 13 depicts the distribution of central atoms with different $\mathrm{CN}$ in the system. 


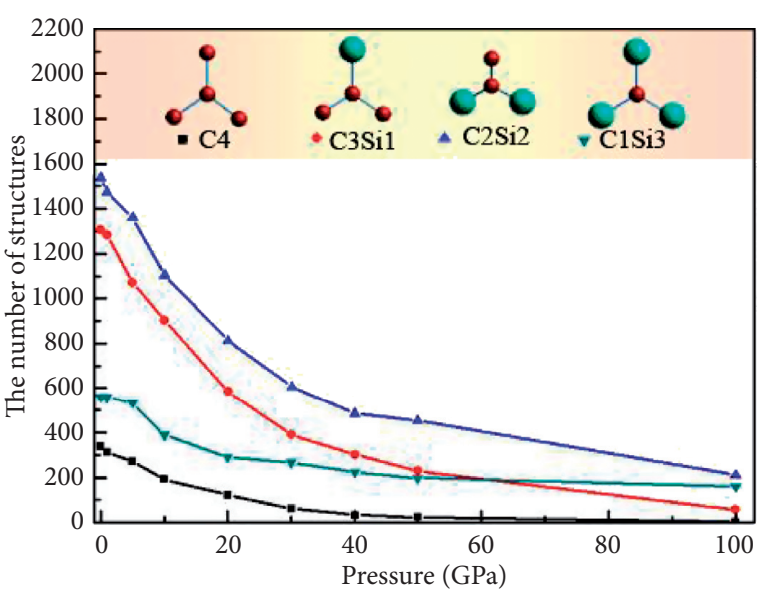

(a)

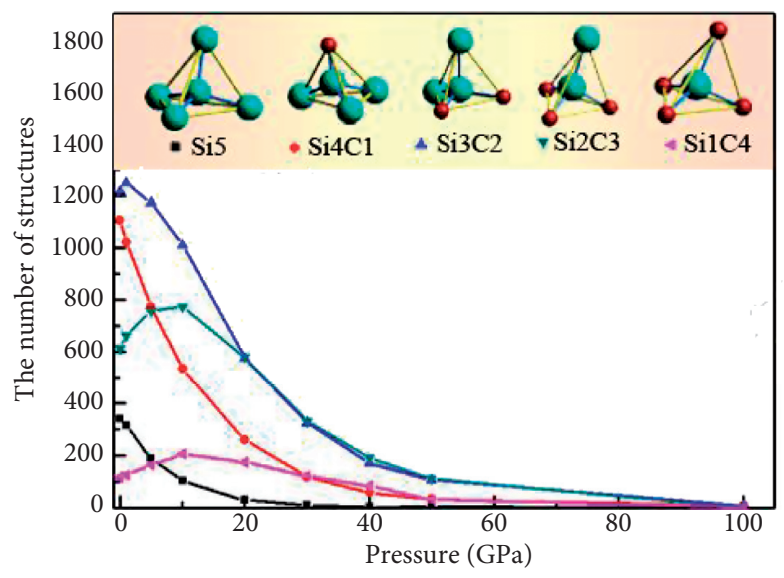

(c)

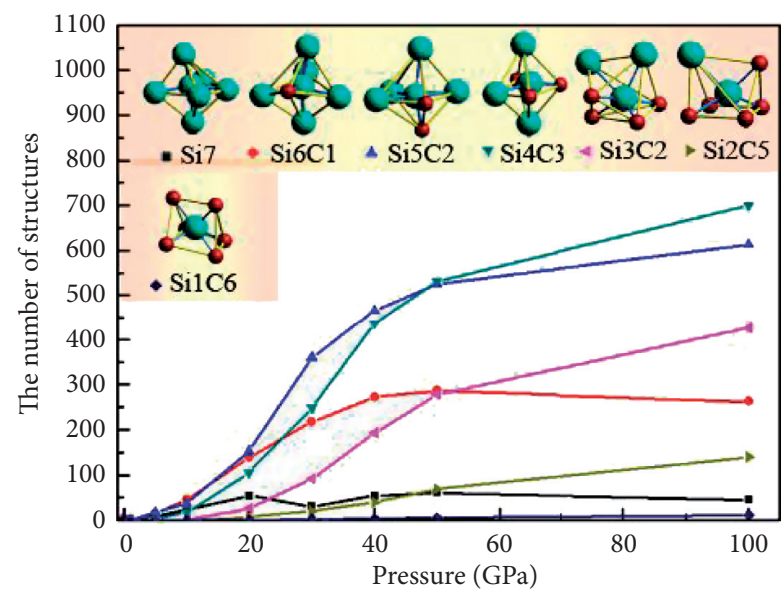

(e)

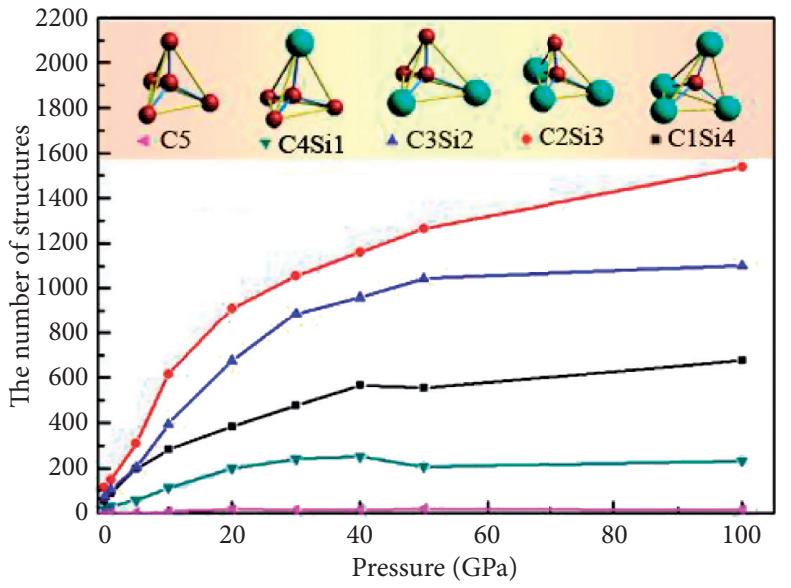

(b)

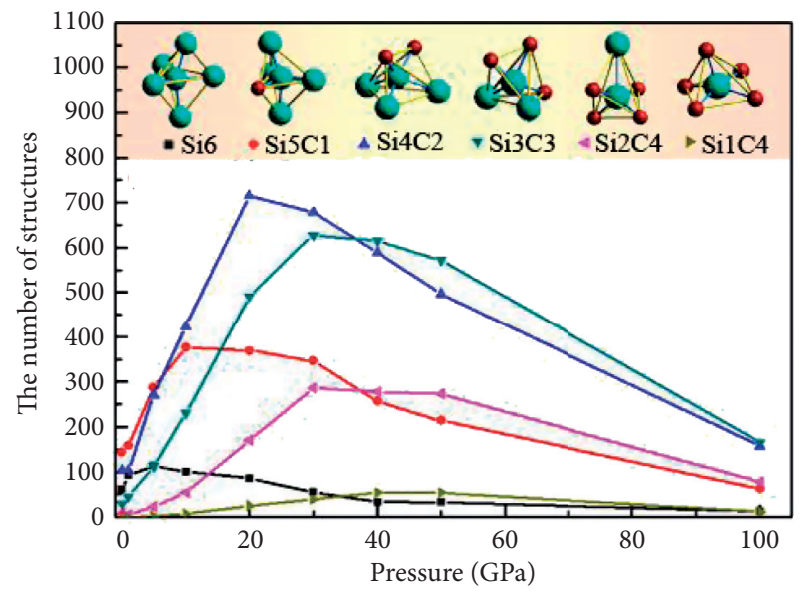

(d)

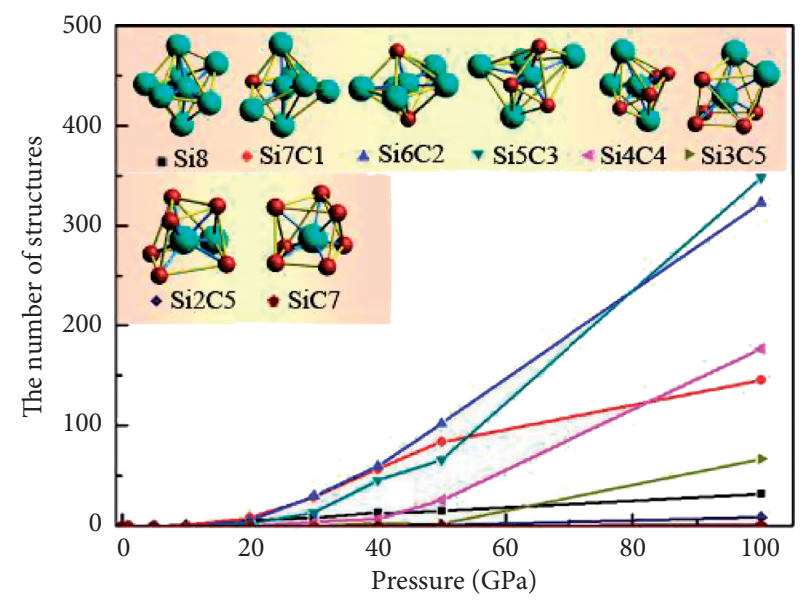

(f)

FIgURE 14: Pressure-dependent changes in the main structure with $\mathrm{C}$ and Si atoms as the central atom (100 K).

3.6.4. Pressure-Dependent Changes in the Main Structure with Central Atom. To analyze the microstructure images of $\mathrm{SiC}$ after solidification under different pressures, the temperature-dependent changes in the four-, five-, six-, and seven-coordination structures of $\mathrm{Si}$ and the three- and fourcoordination structures of $\mathrm{C}$, which are dominant in the rapid solidification process, are analyzed.
AmBn was used to represent the type and the number of atoms in the structure. For example, C1Si3 represents a structure composed of $1 \mathrm{C}$ and $3 \mathrm{Si}$. cn_C_3 represents a three-coordinated structure with $\mathrm{C}$ atoms at the center, respectively. Figure 14 shows the pressure-dependent changes in the main structure with $\mathrm{C}$ or $\mathrm{Si}$ atoms as the central atom at $100 \mathrm{~K}$. 
It can be seen from Figure 14(a) that the number of three-coordinated structures with the $\mathrm{C}$ central atom decreases with increasing pressure, which corresponds to the decrease in the peak value of the $\mathrm{C}-\mathrm{C}$ bond at $\sim 1.5 \AA$ shown in Figure 4(b). It also corresponds to the decrease in the peak value of the bond angle distribution function of cn3_C at $\sim 120^{\circ}$, as shown in Figures $8(\mathrm{a})$. From Figure $14(\mathrm{~b})$, it can be seen that the number of four-coordinated structures with the $\mathrm{C}$ atom at the center increases with increasing pressure, which corresponds to the increase in the bond angle distribution function of cn4_C at $\sim 110^{\circ}$, as shown in Figure 8(b). Furthermore, it can be seen from Figure 14(c) that the number of four-coordinated structures with the $\mathrm{Si}$ atom at the center decreases with increasing pressure, which corresponds to the decrease of the Si-Si bond peak at $\sim 2.35 \AA$ in Figure $4(\mathrm{~d})$ and corresponds to the decrease of the BADF of cn4_Si at $\sim 110^{\circ}$ in Figure $8(\mathrm{c})$. It can be seen from Figure 14(d) that the number of five-coordinated structures with $\mathrm{Si}$ atoms at the center increases first and then decreases with increasing pressure. The number of Si4C2 structures, which is the largest number in cn_Si_5, reaches the maximum value at a pressure of $20 \mathrm{GPa}$, and then gradually decreases with increasing pressure. The number of $\mathrm{Si} 3 \mathrm{C} 3$ and $\mathrm{Si} 2 \mathrm{C} 4$ reached the maximum value at $30 \mathrm{GPa}$, and then gradually decrease. Thus, the change in the five-coordination structure corresponds to the decrease in the peak value of the bond angle distribution function of cn5_Si at $70^{\circ}-120^{\circ}$, as shown in Figure $8(\mathrm{~d})$, and it also corresponds to the change in the number of five-coordination (2 300 ) structures with pressure in Figure 10. From Figures 14(e) and 14(f), it can be seen that the number of six-coordinated and seven-coordinated structures with $\mathrm{Si}$ atoms at the center increases with increasing pressure, which corresponds to the peak increase of the BADF of cn6_Si and cn7_Si in Figures 8(e) and $8(\mathrm{f})$ at $60^{\circ}-180^{\circ}$, and it also corresponds to the change in the number of sixcoordinated ( $\left(\begin{array}{llll}0 & 6 & 0 & 0\end{array}\right)$ and seven-coordinated ( $\left.\begin{array}{llll}0 & 5 & 2 & 0\end{array}\right)$ with pressure in Figure 10.

\section{Conclusion}

From the results of the analysis of the rapid solidification process of liquid $\mathrm{SiC}$ under different pressures, the main conclusions obtained are as follows:

During the solidification process, the application of pressure has a major impact on the arrangement of atoms, except on the third-nearest neighbor. Furthermore, the impact on the nearest neighbor and the next nearest neighbor is small. The increase in pressure makes the atomic arrangement of the system more compact and improves the medium-range order of the system. At higher pressures, the system exhibits a complex bond angle distribution, with three main peaks around $60^{\circ}, 80^{\circ}$, and $110^{\circ}$ and a shoulder peak at $140^{\circ}-180^{\circ}$. The pressure changes the microstructure of the $\mathrm{SiC}$ amorphous system after solidification, and the density is improved by adjusting the $\mathrm{CN}$ atoms. As the pressure increases, the $\mathrm{SiC}$ amorphous system exhibits a dense structure with a $\mathrm{CN}$ of $4,5,6$, and 7 .

\section{Data Availability}

The underlying data supporting the results of our study are submitted along with the manuscript.

\section{Conflicts of Interest}

The authors declare that they have no conflicts of interest.

\section{Acknowledgments}

This work was supported by the National Natural Science Foundation of China (Grant no. 51671004) and the funds from the Key Laboratory of Materials Simulation and Computing of Anshun University (Grant no. Asxyxkpt201803). The authors thank LetPub (https://letpub. com) for its linguistic assistance during the preparation of this manuscript.

\section{References}

[1] Y. T. Yang, K. L. Ekinci, X. M. H. Huang et al., "Monocrystalline silicon carbide nanoelectromechanical systems," Applied Physics Letters, vol. 78, no. 2, pp. 162-164, 2001.

[2] H. yue, J. Peng, and Y. Yang, Silicon Carbide Wide Band Gap Semiconductor Technology, Science Press, Beijing, China, 2000.

[3] M. Yoshida, A. Onodera, M. Ueno, K. Takemura, and O. Shimomura, "Pressure-induced phase transition in SiC," Physical Review B, vol. 48, no. 14, pp. 10587-10590, 1993.

[4] K. K. Zhuravlev, A. F. Goncharov, S. N. Tkachev, P. Dera, and V. B. Prakapenka, "Vibrational, elastic, and structural properties of cubic silicon carbide under pressure up to 75 GPa: implication for a primary pressure scale," Journal of Applied Physics, vol. 113, no. 11, pp. 113503-113511, 2013.

[5] K. Daviau and K. K. M. Lee, "Zinc-blende to rocksalt transition in SiC in a laser-heated diamond-anvil cell," Physical Review B, vol. 95, no. 13, p. 134108, 2017.

[6] K. Daviau and K. K. M. Lee, "Decomposition of silicon carbide at high pressures and temperatures," Physical Review $B$, vol. 96, no. 17, Article ID 174102, 2017.

[7] S. J. Tracy, R. F. Smith, J. K. Wicks et al., "In situ observation of a phase transition in silicon carbide under shock compression using pulsed x-ray diffraction," Physical Review B, vol. 99, no. $21,2019$.

[8] M. Tang and S. Yip, "Atomistic simulation of thermomechanical properties of $\beta$-SiC," Physical Review B, vol. 52, no. 21, pp. 15150-15159, 1995.

[9] C. Koitzsch, D. Conrad, K. Scheerschmidt, and U. Gösele, "Empirical molecular dynamic study of $\mathrm{SiC}(0001)$ surface reconstructions and bonded interfaces," Journal of Applied Physics, vol. 88, no. 12, pp. 7104-7109, 2000.

[10] D. Stoltz, S. E. Stoltz, S. M. Widstrand, and L. S. O. Johansson, "Investigation of surface structure related features in the multiple-scattering simulations of photoelectron diffraction of 3C-SiC (001)-c $(4 \times 2)$," Physica B: Condensed Matter, vol. 395, no. 1, pp. 130-137, 2007.

[11] A. Chatterjee, A. Bhat, and K. Matocha, "Investigation of electrically active defects of silicon carbide using atomistic scale modeling and simulation," Physica B: Condensed Matter, vol. 401, pp. 81-84, 2007.

[12] N. G. Zhou Nai-Gen, T. Hong Tao, and L. Zhou Lang, "A comparative study between MEAM and Tersoff potentials on 
the characteristics of melting and solidification of carborundum," Acta Physica Sinica, vol. 61, no. 2, Article ID 028101, 2012.

[13] H. M. Pan and X. Y. Si, "Molecular dynamics simulations of diameter dependence tensile behavior of silicon carbide nanotubes," Physica B: Condensed Matter, vol. 404, no. 12, pp. 1809-1812, 2009.

[14] Y. Zhang and H. Huang, "Stability of single-wall silicon carbide nanotubes -molecular dynamics simulations," Computational Materials Science, vol. 43, no. 4, pp. 664-669, 2008.

[15] Z. G. Wang, J. B. Li, F. Gao, and W. J. Weber, “Tensile and compressive mechanical behavior of twinned silicon carbide nanowires," Acta Materialia, vol. 58, no. 6, pp. 1963-1971, 2010.

[16] J. Wang, C. Lu, Q. Wang et al., "Influence of microstructures on mechanical behaviours of $\mathrm{SiC}$ nanowires: a molecular dynamics study," Nanotechnology, vol. 23, no. 2, Article ID 025703, 2012.

[17] S. N. Luo, A. Strachan, and D. C. Swift, "Nonequilibrium melting and crystallization of a model lennard-jones system," The Journal of Chemical Physics, vol. 120, no. 24, pp. 11640-11649, 2004.

[18] P. S. Branicio, A. Nakano, R. K. Kalia, and P. Vashishta, "Shock loading on AlN ceramics: a large scale molecular dynamics study," International Journal of Plasticity, vol. 51, no. 6, pp. 122-131, 2013.

[19] W. Li, E. N. Hahn, P. S. Branicio et al., "Rate dependence and anisotropy of $\mathrm{SiC}$ response to ramp and wave-free quasi-isentropic compression," International Journal of Plasticity, vol. 138, 2021.

[20] W. Li, E. N. Hahn, P. S. Branicio, X. Yao, and T. C. Germann, "Effect of Temperature on solidification rate during directional solidification of multicrystaline silicon," Materials Reports, vol. 33, no. Z1, pp. 28-32, 2019.

[21] C. W. Chan and F. L. Tan, "Solidification inside a sphere-an experimental study," International Communications in Heat and Mass Transfer, vol. 33, no. 3, pp. 335-341, 2006.

[22] X. Liu, P. Ma, Y. D. Jia et al., "Solidification of Al-xCu alloy under high pressures," Journal of Materials Research and Technology, vol. 9, no. 3, pp. 2983-2991, 2020.

[23] J. Tersoff, "Modeling solid-state chemistry: interatomic potentials for multicomponent systems," Physical Review B, vol. 39, no. 8, pp. 5566-5568, 1989.

[24] S. Nosé, "A unified formulation of the constant temperature molecular dynamics methods," Journal of Chemical Physics, vol. 81, no. 1, pp. 511-519, 1984.

[25] W. G. Hoover, "Canonical dynamics: equilibrium phase-space distributions," Physical Review A, vol. 31, no. 3, pp. 1695-1697, 1985.

[26] W. J. Yan, T. H. Gao, and X. T. Guo, "Structural properties of liquid SiC during rapid Solidification," The Scientific World Journal, vol. 2013, Article ID 273023, 7 pages, 2013.

[27] J. L. Finney, "Random packings and the structure of simple liquids I. The geometry of random close packing," Proceedings of the Royal Society of London A Mathematical and Physical Sciences, vol. 319, no. 1539, pp. 479-493, 1970. 